\title{
Effect of iontophoresis with growth factor cocktail containing fibroblast growth factor 5-short applied to the scalp in patients with androgenetic alopecia - A split study
}

\author{
Byung In Ro ${ }^{1 *}$, Sung Min Kim ${ }^{1}$, Jae Won Kang ${ }^{1}$ and Hang Cheol Shin ${ }^{2}$ \\ ${ }^{1}$ Department of Dermatology, Myongji Hospital, Hanyang University College of Medicine, Goyang, Korea \\ ${ }^{2}$ School of Systems Biomedical Science, Soongsil University, Seoul, Korea
}

\begin{abstract}
Background: Growth factor cocktail (GFC) in combination with microneedling is an effective and safe treatment for patients with androgenetic alopecia (AGA). However, few studies have been conducted on the absorption of GFC into the scalp through iontophoresis.

Objective: The aim of this study was to evaluate the effect of iontophoresis with GFC, including fibroblast growth factor 5-short (FGF5s), on hair growth in AGA patients.

Methods: The study was performed on AGA patients who were treated with a topical solution and iontophoresis once per day for 24 weeks. The scalp was divided into right and left sides, and treated with GFC including FGF5s (right side) and normal saline (left side). Iontophoresis was performed on the scalp using the headset for 15 min daily. In total, 21 patients (10 men and 11 women) were enrolled. Treatment efficacy was evaluated through analyses of phototrichograms and digital photographs every 4 weeks for 24 weeks.
\end{abstract}

Results: Phototrichogram images showed that 24 weeks of treatment with GFC including FGF5s with iontophoresis increased hair density from $179.2 \pm 5.5 / \mathrm{cm}^{2}$ to $193.9 \pm 5.3 / \mathrm{cm}^{2}(p<0.0001)$ and hair diameter from $54.9 \pm 2.3 \mu \mathrm{m}$ to $55.3 \pm 2.3 \mu \mathrm{m}(p<0.0565)$. The phototrichogram images of the region treated with saline after 24 weeks were not significantly different from baseline in hair density.

Conclusion: Absorption of GFC including FGF5s following iontophoresis was effective for patients with AGA in a time frame of 12 weeks. However, further study is needed on the long term efficacy of absorption of GFC through iontophoresis.

\section{Introduction}

Androgenetic alopecia is the most common hair loss disease; however, insufficient treatments have been established.

Therefore, various treatments are being developed and applied in addition to the use of Food and Drug Administration-approved treatments, such as finasteride and topical minoxidil. Growth factor cocktail (GFC) is also an effective treatment for AGA, and GFC with microneedling is more effective than GFC alone [1]. Microneedling is an effective treatment for AGA because it stimulates hair growth and facilitates drug delivery [2]. We evaluated the effects of scalp penetration via iontophoresis using GFC including FGF5s.

\section{Materials and methods}

\section{Patients}

Twenty-one patients who were diagnosed with mild-to-moderate AGA at the Alopecia Clinic of Department of Dermatology, Hanyang University Medical Center, Myongji Hospital, Goyang, Korea, between October 2018 and May 2019 inclusive, were enrolled. The exclusion criteria for this study included receipt of treatment for AGA within the previous year and having an infectious disease, immunodeficiency, or a history of keloids. This study was approved by the Institutional Review Board of Myongji Hospital, and was performed in accordance with the guidelines of the Declaration of Helsinki.

\section{Materials and treatment regimens}

All subjects underwent treatment with GFC including FGF5s, applied by using an iontophoresis headset once per day for 24 weeks. The scalp was divided into the right and left sides, to which separate treatments were applied: GFC including FGF5s to the right side; and normal saline to the left side. Iontophoresis was applied to the scalp via the headset every $15 \mathrm{~min}$ per day and no other treatments (e.g., topical minoxidil solution or systemic administration of finasteride or dutasteride) were administered.

1) GFC

The GFC used in this study (Cellcurin ${ }^{\mathrm{mw}}$; PnP Biopharm, Seoul, Korea) consisted of basic fibroblast growth factor (bFGF, $3.125 \mu \mathrm{g} / \mathrm{mL}$ ), vascular endothelial growth factor (VEGF, $3.125 \mu \mathrm{g} / \mathrm{mL}$ ), keratinocyte

${ }^{\star}$ Correspondence to: Byung In Ro, Invited Professor, Department of Dermatology, Myongji Hospital, Hanyang University College of Medicine, Goyang 10475, Korea, Tel: +82-31-810-7050; Fax: +82-31-810-7057; E-mail: drro@mjh.or.kr

Key words: androgenetic alopecia, fibroblast growth factor 5-short, growth factor cocktail, iontophoresis

Received: February 19, 2020; Accepted: March 05, 2020; Published: March 10, 2020 
Ro BI (2020) Effect of iontophoresis with growth factor cocktail containing fibroblast growth factor 5-short applied to the scalp in patients with androgenetic alopecia - A split study

growth factor-2 (KGF-2, $3.125 \mu \mathrm{g} / \mathrm{mL})$, stem cell factor (SCF, 3.125 $\mu \mathrm{g} / \mathrm{mL}$ ), insulin-like growth factor-1 (IGF-1, $1.25 \mu \mathrm{g} / \mathrm{mL}$ ), superoxide dismutase-1 (SOD-1, $6.25 \mu \mathrm{g} / \mathrm{mL})$, noggin peptide $(10 \mu \mathrm{g} / \mathrm{mL})$, fibroblast growth factor 9 (FGF9, $3.125 \mu \mathrm{g} / \mathrm{mL}$ ) and fibroblast growth factor 5-short (FGF5s, $10 \mu \mathrm{g} / \mathrm{mL}$ ).

\section{2) Iontophoresis}

The headset consisted of a helmet with a removable pads and a wristband controller operated by a $3 \mathrm{~V}$ battery. It was set to apply treatment for $15 \mathrm{~min}$ (Figure 1).

\section{3) Treatment}

GFC including FGF5s was provided as a liquid. Approximately 2.5 $\mathrm{mL}$ of the GFC solution was topically applied on the right side of the scalp, and $2.5 \mathrm{~mL}$ of normal saline on the left side of the scalp (Figure 2). Treatment was applied to each side of the scalp by using an iontophoresis headset once per day for 24 weeks. The daily iontophoresis treatment was applied to the entire head for 15 min every day.

\section{Measurement of parameters}

To ensure reproducibility, a tattoo point was placed on the right and left sides of the scalp of each patient before treatment. A phototrichogram (Folliscope 2.8; Lead M, Seoul, Korea) was taken from a fixed area marked by the tattoo on each sides to measure hair density and the diameter at the baseline and at the end of treatment. An investigator blinded to the study counted the number of hairs and the hair diameter from the phototrichogram images.

\section{Statistical analysis}

11 data were expressed as the mean \pm standard deviation. Wilcoxon matched-pairs signed-rank test was computed by using STATA/ SE ver.12 (Stata Corp LP, College Station, TX, USA) to evaluate the effectiveness of treatment on each patient. Student's $t$-test $(p$-value $<0.05)$ was performed to analyze the difference between the effect of treatment applied to the right and left sides of the scalp.

\section{Results}

\section{Patient characteristics}

Twenty-one patients, from 24 to 45 years of age (mean, $34.5 \pm 7.4$ years of age) completed the 6-month study. The patients comprised: 10 men, with a mean age of $38.7 \pm 7.2$, and male pattern hair loss type IIa (1 patient) type III (4 patients), type IIIv (4 patients), and type IV (1 patient) according to the Norwood-Hamilton classification; and 11 were women, with a mean age of $30.8 \pm 5.4$ years, and female pattern hair loss type I (7 patients) and type II (4 patients) according to the Ludwig scale (Table 1).
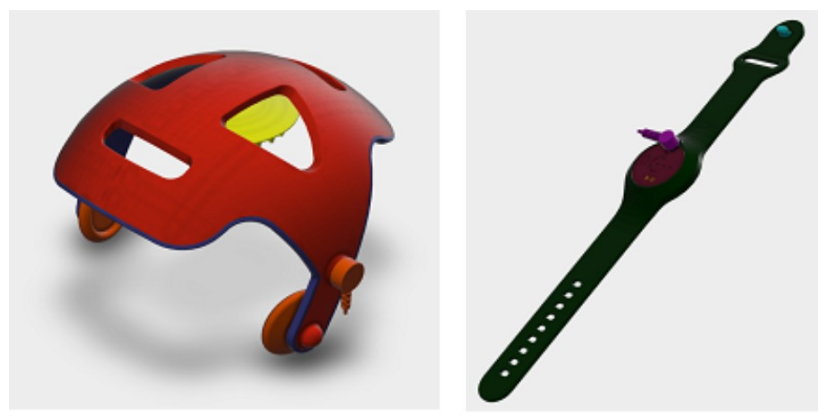

Figure 1. The headset model consists of a helmet with a removable pads and a wristband controller

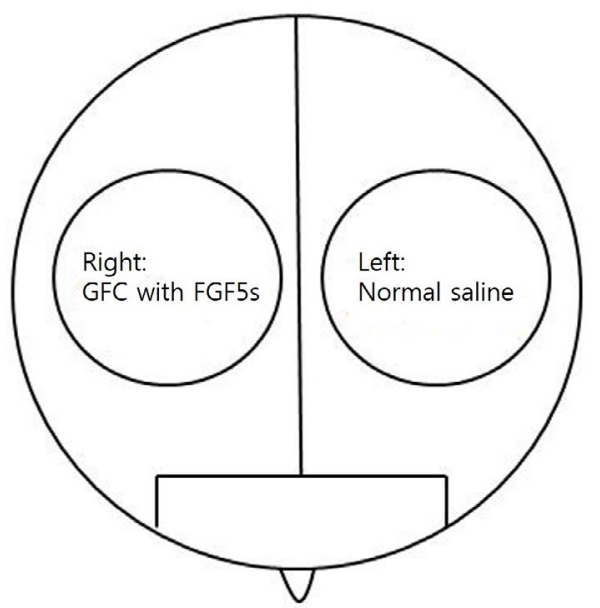

Figure 2. Schematic diagram of the division of the scalp into right and left sides for the split test

Table 1. Baseline characteristics of the study patients

\begin{tabular}{|c|c|c|c|}
\hline & Total & Men & Women \\
\hline Patients & 21 & 10 & 11 \\
\hline Mean age & $34.5 \pm 7.4$ & $38.7 \pm 7.2$ & $30.8 \pm 5.4$ \\
\hline \multicolumn{4}{|c|}{ Pattern hair loss } \\
\hline MPHL IIa & - & 1 & - \\
\hline MPHL III & - & 4 & - \\
\hline MPHL IIIv & - & 4 & - \\
\hline MPHL IV & - & 1 & - \\
\hline FPHL I & - & - & 7 \\
\hline FPHL II & - & - & 4 \\
\hline
\end{tabular}

MPHL: Male pattern hair loss; FPHL: Female pattern hair loss.

\section{Treatment efficacy and adverse effects}

Split test was conducted to determine the efficacy of GFC including FGF5s in patients with AGA. The patient's scalp was divided into the right and left sides: both were treated with iontophoresis; however, the right side was treated with GFC and the left side was treated with normal saline with iontophoresis. The density and diameter of hair were measured at baseline and after 24 weeks. The phototrichogram images showed that the treatment increased the density of hair on the right side (GFC) and the diameter of hair on the left side (normal saline) of the scalp (Figure 2). Increased growth was observed on the right side of the scalps treated with GFC, which included FGF5s (Figure 3). The changes in the density and diameter of hair between the right and left sides after 24 weeks of treatment are shown in Table 2. Phototrichogram images showed that 24 weeks of treatment with GFC including FGF5s and iontophoresis increased hair density from $179.2 \pm 5.5 / \mathrm{cm}^{2}$ to $193.9 \pm 5.3 /$ $\mathrm{cm}^{2}(p<0.0001)$ and hair diameter from $54.9 \pm 2.3 \mu \mathrm{m}$ to $55.3 \pm 2.3 \mu \mathrm{m}$ $(p<0.0565)$. The phototrichogram images of the region treated with saline after 24 weeks were not significantly different from baseline in both hair density and diameter. There were no adverse reactions related to the treatment. These results were evaluated after consultation with two hair specialists in other hospitals (Figure 4).

\section{Discussion}

AGA, also known as androgenic alopecia or male pattern hair loss, is the most common type of progressive hair loss. Patients who have prominent thinning of the hair are perceived as older, which affects self-esteem and leads to psychosocial morbidity [3]. Although many 


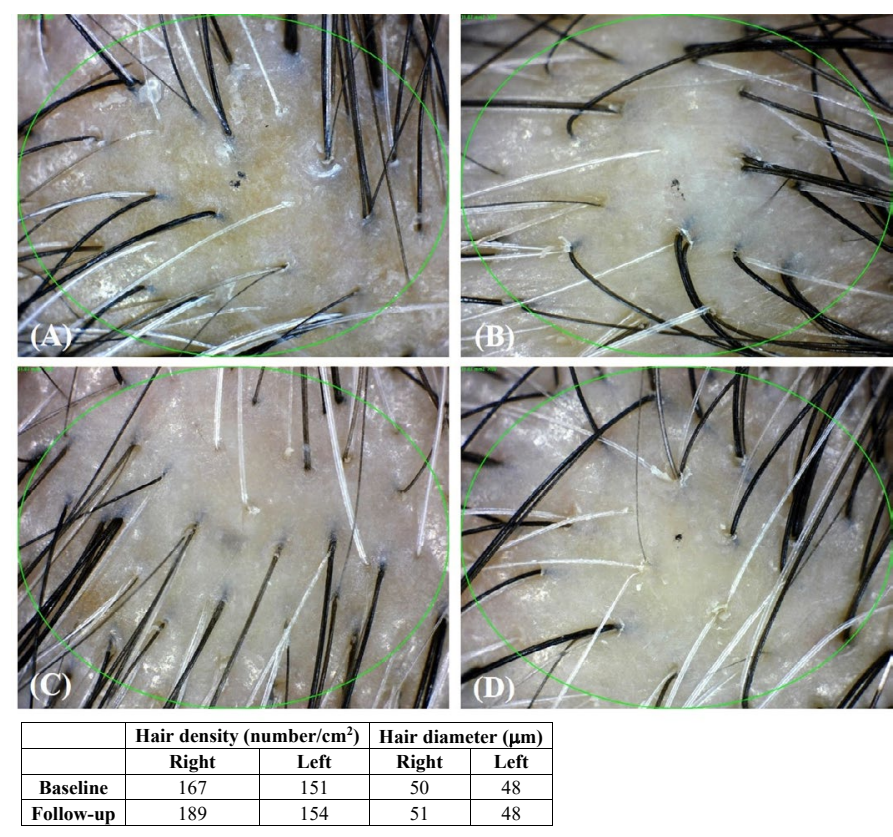

Figure 2. Schematic diagram of the division of the scalp into right and left sides for the split test

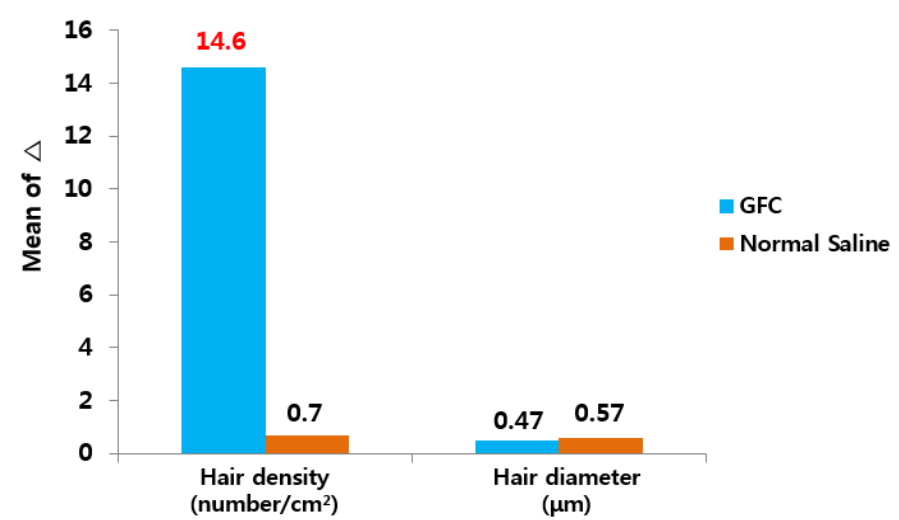

Figure 3. Evaluation of phototrichogram images after treatment for 24 weeks (A: Baseline, right side of the scalp; B: Baseline, left side of the scalp; C: After 24 weeks, right side of the scalp; D: After 24 weeks, left side of the scalp)

Table 2. Statistical analysis of the changes in hair density and diameter between baseline and at 24 weeks after treatment

\begin{tabular}{|c|c|c|c|c|c|}
\hline & Baseline & 24 weeks & & \\
\hline Hair density & GFC & $179.2 \pm 5.5$ & $193.9 \pm 5.3$ & $14.6 \pm 1.4$ & $<$-Value \\
\hline $\begin{array}{c}\text { (number/ } \\
\left.\mathbf{c m}^{2}\right)\end{array}$ & $\begin{array}{c}\text { Normal } \\
\text { saline }\end{array}$ & $167.1 \pm 4.8$ & $167.8 \pm 4.7$ & $0.7 \pm 0.35$ & $<0.0001$ \\
\hline $\begin{array}{c}\text { Hair } \\
\text { diameter }\end{array}$ & GFC & $54.9 \pm 2.3$ & $55.3 \pm 2.3$ & $0.47 \pm 0.24$ & 0.0565 \\
\hline $\mathbf{( \mu m )}$ & $\begin{array}{c}\text { Normal } \\
\text { saline }\end{array}$ & $53.85 \pm 2.33$ & $54.43 \pm 2.25$ & $0.57 \pm 0.23$ & 0.0244 \\
\hline
\end{tabular}

GFC: Growth factor cocktail; SD: Standard deviation; $\Delta$ : Change.

options are available to treat AGA, the medications approved by the Food and Drug Administration are limited to oral finasteride and topical minoxidil. Therefore, new options, such as stem cell therapy and recombinant growth factor techniques [4], continue to be evaluated for safe and effective treatments. Through research into various cytokines that contribute to hair growth and differentiation, biochemical therapies are emerging as new options for AGA treatment. The growth factors studied in relation to hair follicle growth include vascular endothelial growth factor (VEGF), epidermal growth factor (EGF), insulin like growth factor-1 (IGF-1) beta-fibroblast growth factor (bFGF), wingless-related integration site (Wnt), noggin, keratinocyte growth factor, and copper tripeptides. These growth factors may be safe, cheap, and nonallergenic tools for the management of alopecia [5-7].

Hair follicles in the anagen phase contain several growth factors, such as IGF-1, EGF, FGFs, and Platelet-derived growth factor (PDGF), which bind with the relevant tyrosine kinase receptors and are activated through two signaling pathways-the Ras-Raf-MEK-ERK pathway and the phosphatidylinositol-3-kinase (PI3K)-PDK1-Akt pathway; the Ras pathway is known to regulate cell migration and proliferation, survival, differentiation, and senescence [8]. Of the various growth factors, FGF9, initially secreted by $\mathrm{T}$ cells, modulates hair follicle regeneration following induction of a skin wound in adult mice. FGF9 from $\mathrm{T}$ cells triggers Wnt expression and subsequent Wnt activation in wound fibroblasts, and has been reported to be a triggering factor for Wnt expression and a hair follicle regenerator [9]. Recently, our group reported that GFC including FGF9 was effective for improving hair growth and thickening on patients with pattern hair loss in clinical study [10].

The treatment solution used in this study contained bFGF, VEGF, KGF-2, SCF, IGF-1, SOD-1, noggin peptide, FGF9, and FGF5s as the main ingredients. These factors can promote the cell cycle, regenerate hair cells, and protect hair follicles from apoptotic signals.

Xiaolin He et al. [11] reported that FGF5s inhibits function of FGF5, which regulates the transition from anagen to catagen phase in dermal papilla cells of cashmere goats. FGF5 is a key determinant of the anagen:telogen ratio, and hair length, in human hair follicles. In addition, FGF5 acts as a stimulus for catagen entry, by binding to Fibroblast growth factor receptor1 (FGFR1) located within the dermal papilla, a mesenchymal signaling center located at the base of the hair follicle. FGF5s introduction can antagonize this effect [12], presumably by competitively binding the FGF5 receptor, FGFR1 [13]. Overexpression of FGF5 suppressed the mRNA expression of IGF-1, versican, and noggin, but enhanced bone morphogenetic protein 4 in primary hair follicles and secondary hair follicles - dermal papilla cells of cashmere goats [11]. FGF5s overexpression attenuated the FGF5-induced changes in expression in these genes [11]. In this study, we have more clearly identified the significant increase in hair count resulting from treatment with GFC containing FGF5s.

In the past, GFC has been injected intracutaneously using microneedles; recently, various methods have been used to increase permeation efficiency, including mesotherapy, diode laser, and microneedle roller.

Kapoor R et al. [14] reported that the intradermal injection of hair growth formulation resulted in a significant increase in total hair count in patients with male pattern hair loss and with female pattern hair loss. However, these injection methods may be accompanied by pain, the most common side effect; therefore, various methods have been considered to solve this problem.

In recent years, many studies have been reported on the high efficiency of intradermal permeation via iontophoresis. There are three proposed mechanisms for transdermal delivery through iontophoresis [15]. The first proposes that the drug is forced across the skin by simple 
Ro BI (2020) Effect of iontophoresis with growth factor cocktail containing fibroblast growth factor 5-short applied to the scalp in patients with androgenetic alopecia - A split study

electronic repulsion of similar charges. The second suggests that the electric current enhances the permeation through an inhibition of the skin's ability to perform its protective barrier function. The third states that iontophoresis causes water, a very effective penetration enhancer, to enter the stratum corneum by electroosmosis.

In our study, the count of hairs was increased significantly at the site of injection of GFC including FGF5s on the scalp by iontophoresis compared with that at the site treated with normal saline. We found that each protein component of our GFC had a different isoelectric point. Based on this, the iontophoresis headset was designed to produce an alternating current rather than a direct current, to obtain more efficient scalp penetration of every protein component.

he limitations of this study were the relatively small number of enrolled patients. The iontophoresis headset used in our study was made in a uniform size. If the headset was made in various sizes to contact with the scalp more tightly, more significant results may be obtained. In addition, unlike conventional studies of the effect of other components of iontophoresis on the skin (e.g., vitamin C), the thick scalp skin and many hairs made it hard to apply the effects of iontophoresis directly.

\section{Conclusion}

In this study, we have provided the first evaluation of the effect of iontophoresis after the application of GFC to the scalp. In addition, we tried to find a clearer using GFC that included FGF5s, which was recently identified to delay the growth phase. As a result, the number of hair counts was significantly increased after infiltration through iontophoresis of GFC including FGF5s. Hence, iontophoresis could be another alternative to the GFC injection in the future, as it can be applied more easily by itself than conventional injection methods that cause pain or are reliant on the clinician's skill.

\section{Conflict of interest}

No potential conflict of interest relevant to this article is reported.

\section{Acknowledgments}

Cellcurin $^{\text {twe }}$ was provided by PNP Biopharm (Seoul, Korea). Korea).
"This research (MJH 16-069) was performed as part of the Research Program for Clinical Professor of Myongji Hospital, Goyang 10475, Korea.

\section{References}

1. Lee YB, Eun YS, Lee JH, Cheon MS, Park YG, et al. (2013) Effects of topical application of growth factors followed by microneedle therapy in women with female pattern hair loss: A pilot study. J Dermatol 40: 81-83. [Crossref]

2. Chandrashekar B, Yepuri V, Mysore V (2014) Alopecia areata-successful outcome with micro needling and triamcinolone acetonide. J Cutan Aesthet Surg 7: 63-64. [Crossref]

3. Han SH, Byun JW, Lee WS, Kang H, Kye YC, et al. (2012) Quality of life assessment in male patients with androgenetic alopecia: result of a prospective, multicenter study. Ann Dermatol 24: 311-318. [Crossref]

4. Varothai S, Bergfeld WF (2014) Androgenetic alopecia: An evidence-based treatment update. Am J Clin Dermatol 15: 217-230. [Crossref]

5. Price VH (1999) Treatment of hair loss. N Engl J Med 341: 964-973. [Crossref]

6. Ebling FG, Hale PA, Randall VA (1991) Hormones \& hair growth. In: Goldsmith LA editor. Biochemistry \& physiology of the skin. 2nded. Clarendon Press, Oxford, pp: 660-690.

7. Parsley WM, Perez-Meza D (2010) Review of factors affecting the growth\& survival of follicular grafts. J Cutan Aesthet Surg 3: 69-75. [Crossref]

8. Rishikaysh P, Dev K, Diaz D, Qureshi WM, Filip S, et al. (2014) Signaling involved in hair follicle morphogenesis and development. Int J Mol Sci 15: 1647-1670. [Crossref]

9. Gay D, Kwon O, Zhang Z, Spata M, Plikus MV, et al. (2013) Fgf9 from dermal $\gamma \delta \mathrm{T}$ cells induces hair follicle neogenesis after wounding. Nat Med 19: 916-923. [Crossref]

10. Ro BI, Chun SW, Lee SY, Shin HC (2016) Therapeutic effects of growth factor cocktail including fibroblast growth factor 9 in patients with androgenetic alopecia - a split study. Glob Dermatol 3: 404-408.

11. He X, Chao T, Zhou G, Chen Y (2015) Fibroblast growth factor 5-short (FGF5s) inhibits the activity of FGF5 in primary and secondary hair follicle dermal papilla cells of cashmere goats. Gene 575: 393-398. [Crossref]

12. Suzuki S, Ota Y, Ozawa K, Imamura T (2000) Dual-mode regulation of hair growth cycle by two Fgf-5 gene products. J Invest Dermatol 114: 456-463. [Crossref]

13. Ozawa K, Suzuki S, Asada M, Tomooka Y, Li AJ, et al. (1998) An alternatively spliced fibroblast growth factor (FGF)-5 mRNA is abundant in brain and translates into a partial agonist/antagonist for FGF-5 neurotrophic activity. J Biol Chem 273: $29262-$ 29271. [Crossref]

14. Kapoor R, Shome D (2018) Intradermal injections of a hair growth factor formulation for enhancement of human hair regrowth - safety and efficacy evaluation in a firstin-man pilot clinical study. Journal of Cosmetic and Laser Therapy 20: 369-379. [Crossref]

15. Bharkatiya M, Nema RK (2009) Skin penetration enhancement techniques. $J$ Young Pharm 1: 110-115.

Copyright: (C2020 Ro BI. This is an open-access article distributed under the terms of the Creative Commons Attribution License, which permits unrestricted use, distribution, and reproduction in any medium, provided the original author and source are credited. 